\title{
Complex two-gene modulation of lung disease severity in children with cystic fibrosis
}

Ruslan Dorfman, ${ }^{1}$ Andrew Sandford, ${ }^{2}$ Chelsea Taylor, ${ }^{3}$ Baisong Huang, ${ }^{3}$ Daisy Frangolias, ${ }^{2}$ Yongqian Wang, ${ }^{1}$ Richard Sang, ${ }^{1}$ Lilian Pereira, ${ }^{1}$ Lei Sun, ${ }^{4}$ Yves Berthiaume, ${ }^{5}$ Lap-Chee Tsui, ${ }^{1}$ Peter D. Paré, ${ }^{2}$ Peter Durie, ${ }^{6}$ Mary Corey, ${ }^{3}$ and Julian Zielenski ${ }^{1}$

\begin{abstract}
${ }^{1}$ Program in Genetics and Genome Biology, Hospital for Sick Children, Toronto, Ontario, Canada. ${ }^{2}$ The James Hogg iCAPTURE Centre for Cardiovascular and Pulmonary Research, University of British Columbia, Vancouver, British Columbia, Canada. ${ }^{3}$ Child Health Evaluative Sciences Program, Hospital for Sick Children, Toronto, Ontario, Canada. ${ }^{4}$ Department of Public Health Sciences and Department of Statistics, University of Toronto, Toronto, Ontario, Canada. 5 Department of Medicine, Centre de Recherche Centre Hospitalier de l'Université de Montréal, Montreal, Quebec, Canada. ${ }^{6}$ Program in Physiology and Experimental Medicine, Hospital for Sick Children, Toronto, Ontario, Canada.
\end{abstract}

\begin{abstract}
Although cystic fibrosis (CF) is a monogenic disease, its clinical manifestations are influenced in a complex manner. Severity of lung disease, the main cause of mortality among CF patients, is likely modulated by several genes. The mannose-binding lectin $2(M B L 2)$ gene encodes an innate immune response protein and has been implicated as a pulmonary modifier in CF. However, reports have been conflicting, and interactions with other modifiers have not been investigated. We therefore evaluated the association of MBL2 with CF pulmonary phenotype in a cohort of 1,019 Canadian pediatric CF patients. MBL2 genotypes were combined into low-, intermediate-, and high-expression groups based on MBL2 levels in plasma. Analysis of age at first infection with Pseudomonas aeruginosa demonstrated that MBL2 deficiency was significantly associated with earlier onset of infection. This MBL2 effect was amplified in patients with high-producing genotypes of transforming growth factor beta 1 (TGFB1). Similarly, MBL2 deficiency was associated with more rapid decline of pulmonary function, most significantly in those carrying the high-producing TGFB1 genotype. These findings provide evidence of gene-gene interaction in the pathogenesis of CF lung disease, whereby high TGF- $\beta 1$ production enhances the modulatory effect of MBL2 on the age of first bacterial infection and the rate of decline of pulmonary function.
\end{abstract}

\section{Introduction}

Cystic fibrosis (CF) is an autosomal-recessive disease typically manifesting with progressive obstructive lung disease, insufficiency of the exocrine pancreas, and elevated electrolyte concentration in sweat glands (OMIM 219700). Although CF is a monogenic disease caused by mutations in the CF transmembrane conductance regulator (CFTR) gene, genotype-phenotype correlation studies of some manifestations suggest more complex inheritance, involving other genetic and environmental factors $(1,2)$. In particular, the severity of pulmonary disease, which is the major cause of morbidity and mortality in CF, is poorly correlated with CFTR genotype. Patients carrying the same CFTR mutations show extremely variable lung phenotypes at all ages. It is postulated that the severity and progression of pulmonary disease in $\mathrm{CF}$ is modulated by secondary genetic factors called CF modifiers $(1,3)$.

The hallmark of CF pulmonary disease is chronic infection with characteristic pathogens such as Pseudomonas aeruginosa, Staphylococcus aureus, and Burkholderia cepacia, which leads to chronic inflammatory damage to lung tissue and progressive loss of pulmonary function. Genetic factors that influence susceptibility to infection are therefore of major interest. One of the first genes implicated as a pulmonary modifier in CF was the mannose-binding lectin 2 (MBL2) gene (4). MBL2 produces a protein of the collectin family, a key factor in the innate immune response $(5,6)$. MBL2 selectively binds D-mannose and N-acetyl-D-glucosamine (GlcNAc) on bacte-

Nonstandard abbreviations used: $\mathrm{CF}$, cystic fibrosis; $\mathrm{FEV}_{1}$, forced expiratory volume in $1 \mathrm{~s}$; PI, pancreatic insufficiency.

Conflict of interest: The authors have declared that no conflict of interest exists. Citation for this article: J. Clin. Invest. 118:1040-1049 (2008). doi:10.1172/JCI33754 rial surfaces and triggers MBL2-associated serine proteases (MASPs), activation of the complement pathway, and eventual phagocytosis of opsonized bacteria (7). MBL2 also regulates the secretion of cytokines: intermediate levels of MBL2 $(1-5 \mu \mathrm{g} / \mathrm{ml})$ trigger secretion of proinflammatory cytokines (IL-6, TNF- $\alpha$, and monocyte chemoattractant protein 1), while high levels of MBL2 (>6 $\mu \mathrm{g} / \mathrm{ml})$ are known to repress secretion of proinflammatory cytokines and upregulate the antiinflammatory cytokine IL-10 $(8,9)$. On the one hand, high levels of cytokines are beneficial because they initiate pathways necessary for clearing bacterial infection, but on the other hand, they can stimulate inflammatory cascades that are potentially detrimental to the lung tissue of CF patients (10).

MBL2 protein is known to bind to its substrates exclusively in an oligomerized form, and the collagen-like repeats are essential for the multimerization process. In the general population, levels of oligomerized MBL2 in blood vary dramatically (11), which is partially explained by genetic variation in $M B L 2$. The rare $\mathrm{X}$ allele at position -221 of the promoter results in decreased promoter activity, while the missense mutations in exon 1 at positions 52, 54, and 57 in the collagen-like domain abolish assembly of the protein into its biologically active form (12-14). It is well documented that low levels of MBL2 in the blood are associated with susceptibility to infections in various clinical settings $(15,16)$. Independent studies found 2 -fold increase in childhood infections associated with MBL2 deficiency $(17,18)$.

In some studies of patients with CF, MBL2 deficiency has been associated with more severe pulmonary disease $(4,1-21)$ and poor survival $(4,22)$, while others have reported no association $(23,24)$. These studies were based on relatively small CF patient cohorts (about 100-300). A recent study of a large cohort of CF patients homozygous for the $\Delta \mathrm{F} 508$ mutation found no associa- 
tion between $M B L 2$ variants and pulmonary function (25). The Drumm study was specifically designed to include age-specific extremes of pulmonary function, and patients under 8 years of age were excluded because of inability to define the severity of pulmonary disease. This precluded the ability to test for potential effects of the $M B L 2$ polymorphisms at an early stage of CF lung disease.

TGF- $\beta 1$ is a growth factor that plays a critical role in inflammatory processes likely to have pleiotropic effects in the lung. It prevents spontaneous activation of alveolar macrophages and secretion of proinflammatory cytokines and also causes inflammation, apoptosis, remodeling, and fibrosis of lung tissue, but does not affect the efficiency of phagocytosis (26-30). Several transforming growth factor beta 1 (TGFB1) gene variants were previously shown to affect the level of secreted protein $(31,32)$ and were reported to affect severity of lung disease in CF patients (25). Excessive tissue fibrosis in CF lungs was proposed as the basis underlying the association of the high TGF- $\beta 1$-producing allele $C$ in codon 10 of the gene with severe pulmonary CF disease (25).

There were 3 main objectives of this study. The first was to evaluate MBL2 as a modifier of CF lung pathophysiology - represented by age of acquisition of $P$. aeruginosa and rate of decline of pulmonary function - in a large, representative sample of CF patients and to reconcile different findings reported in previous studies $(4,19,21,25)$. The second was to examine the impact of another well-documented pulmonary CF modifier, TGF- $\beta 1(25,33)$, on these lung-related traits in young patients with CF disease. The final objective was to test for potential comodulation of both lung phenotypes by the MBL2 and TGFB1 genes and to analyze interactions between them.

Accordingly, CF patients in this study were recruited without selection bias and are representative of the general CF population in Canada (34). As the high variance of MBL2 concentrations in blood precluded effective grouping for our analysis, we turned to direct genotyping for the missense mutations and promoter polymorphism in the gene as our measurement of MBL2 activity. We also tested for potential gene-gene interactions between $M B L 2$ and functional polymorphisms in the TGFB1 gene, which were reported to modify the severity of pulmonary CF disease (25).

Our results showed that CF children with MBL2 deficiency acquired $P$. aeruginosa infection at a significantly earlier age and presented with more severe pulmonary disfunction than those with the intermediate or high levels of circulating MBL2. We also demonstrated that these modulatory effects were enhanced by the high-producing TGFB1 gene variants, suggesting a complex gene-gene interaction between MBL2 and TGF- $\beta 1$.

\section{Results}

Demographic and clinical characteristics of the pediatric CF patient cohort. The cohort of 1,019 CF pediatric patients is summarized in Table 1. The largest group of CF patients sharing the same CFTR genotype consists of those homozygous for the $\Delta \mathrm{F} 508$ mutation (35). This severely affected CF group serves as a clinical reference for patients with other CFTR genotypes $(36,37)$. We compared the demographic and clinical parameters in CF patients homozygous for $\Delta F 508$ with the rest of the pancreatic insufficiency (PI) group (Table 1). None of the demographic and clinical parameters were significantly different between the 2 groups.
$M B L 2$ gene variants and genotypes. Four MBL2 gene variants (Table 2) were typed in 1,393 CF patients and 600 randomly selected parents from the entire CF Modifier Study cohort (see Methods). The distributions of these variants and related genotypes and diplotypes in patients and parents are shown in Supplemental Table 2 (supplemental material available online with this article; doi:10.1172/ JCI33754). The overall allelic and genotypic frequencies were similar in the patient and parent cohorts and were not different from the previously published data $(4,11)$. We then established 6 major diplotypes from family members' genotypes at 4 variant sites (Table 3); their frequencies were similar in patients and parents (Supplemental Table 2).

$M B L 2$ protein levels in MBL2 diplotype groups. The plasma concentration of oligomerized MBL2 protein was measured in the 1,393 CF patients and 600 parents for whom the diplotypes had been established. As reported previously $(11,12)$, expression of MBL2 oligomers and $M B L 2$ genotypes were significantly correlated. The quantity of MBL2 protein in both the parent group (Figure $1 \mathrm{~A}$ ) and the CF patient group (Figure 1B) strongly correlated with the 6 diplotype groups (Table 3 ). The normalized MBL2 expression increased with the proportion of $A$ and $Y$ alleles in these diplotypes, from ${ }^{*} 00$ to AOXY, AOYY, AAXX, AAXY, and AAYY (Figure 1). The functional consequences of structural mutations in exon 1 that prevent MBL2 assembly (combined as the 0 allele) were more pronounced than the corresponding promoter variant $\mathrm{X}$ that is associated with lower MBL2 expression. This observation can be explained by the fact that homozygosity for any of the structural mutations or their compound heterozygotes prevents oligomerization of MBL2 (14), while homozygosity for the X allele reduces production of the MBL2 oligomer. However, considerable variation in expression was observed for various diplotypes, except for the ${ }^{* *} 00$ diplotype (Figure 1 , A and B), where the presence of 2 structural mutations (denoted as 00 ) completely prevented oligomerization of MBL2 (14).

The lowest level of MBL2 expression in CF and parent cohorts was associated with 2 inferred diplotypes: ${ }^{* *} 00$ and XYA0. Pairwise analysis demonstrated that MBL2 expression was not statistically different between individuals carrying the ${ }^{* *} 00$ and XYAO diplotypes. Therefore, the low MBL2 expression and MBL2-deficient diplotype groups were combined to form the "low MBL2" dip-

\section{Table 1}

Demographic and clinical characteristics of CF patients (age $<18.5 \mathrm{yr}$ ) with PI

\begin{tabular}{|c|c|c|c|c|}
\hline & \multirow[t]{2}{*}{ All patients } & \multicolumn{2}{|c|}{ By CFTR mutation ${ }^{A}$} & \multirow[t]{2}{*}{$P^{B}$} \\
\hline & & $\Delta \mathrm{F} 508 / \Delta \mathrm{F} 508$ & Other PIC & \\
\hline$n^{\mathrm{D}}$ & 1,019 & 611 & 408 & \\
\hline Mean age (yr) & 10.5 & 10.5 & 10.5 & 0.94 \\
\hline Median age of diagnosis (yr) & 0.36 & 0.35 & 0.38 & $0.35^{\mathrm{E}}$ \\
\hline Meconium ileus & $21.2 \%$ & $22.3 \%$ & $19.6 \%$ & 0.35 \\
\hline CF liver disease & $4.4 \%$ & $3.8 \%$ & $5.4 \%$ & 0.22 \\
\hline CF-related diabetes & $2.8 \%$ & $2.9 \%$ & $2.7 \%$ & 0.85 \\
\hline Mean $\mathrm{FEV}_{1} \%$ predicted & $84.1 \%$ & $83.7 \%$ & $84.8 \%$ & 0.50 \\
\hline P. aeruginosa positive & $69.0 \%$ & $67.3 \%$ & $71.6 \%$ & 0.15 \\
\hline Median age of first infection (yr) & 7.2 & 7.5 & 6.9 & $0.13^{\mathrm{F}}$ \\
\hline B. cepacia positive & $5.5 \%$ & $5.9 \%$ & $4.9 \%$ & 0.57 \\
\hline
\end{tabular}

${ }^{A}$ CFTR mutations for patients in this cohort are listed in Supplemental Table 4. ${ }^{\mathrm{B}} \Delta \mathrm{F} 508 / \Delta \mathrm{F} 508$ versus other $\mathrm{PI}$; calculated by Student $t$ test or Fisher exact test except where noted. CIncludes $\Delta \mathrm{F} 508 /$ other and other/other severe genotypes. DExcept mean $\mathrm{FEV}_{1} \%$ predicted ( $n=801$ total patients, $483 \Delta \mathrm{F} 508 / \Delta \mathrm{F} 508$, and 318 other PI). EWilcoxon rank-sum test. FLog-rank test of time to event. 


\section{Table 2}

$M B L 2$ gene variants and notation in the current study

$\begin{array}{lcccc}\text { Variant } & \text { Location } & \text { Nucleotide variant } & \text { Amino acid change } & \text { rs no. } \\ \text { YA } & \text { Promoter } & -221 \mathrm{G} & - & - \\ \mathrm{X} & \text { Promoter } & -221 \mathrm{C} & - & 7096206 \\ \mathrm{~A}^{\mathrm{A}} & \text { Exon 1 } & \text { 154C, 161G, 170G } & \text { Arg52, Gly54, Gly57 } & - \\ \mathrm{B}^{\mathrm{B}} & \text { Exon 1 } & 154 \mathrm{~T} & \text { Cys52 } & 5030737 \\ \mathrm{C}^{B} & \text { Exon 1 } & 161 \mathrm{~A} & \text { Asp54 } & 1800450 \\ \mathrm{D}^{\mathrm{B}} & \text { Exon 1 } & 170 \mathrm{~A} & \text { Glu 57 } & 1800451\end{array}$

AWild type. BDue to the same structural effect on the MBL2 protein, the B, C, and $D$ variants were combined as the 0 variant group. lotype group (Table 4). Similarly, at the other extreme, the 2 diplotypes corresponding to the highest expression, AAXY and AAYY, were combined into the "high MBL2" diplotype group (Table 4). The remaining 2 diplotypes demonstrating intermediate levels of MBL2 expression, including the rare XXAA diplotype (present in $3.7 \%$ of patients) and the more common diplotype YYA0 (present in $25.8 \%$ of patients), were merged into an "intermediate $M B L 2$ " diplotype group (Table 4). The range of expression of the XXAA diplotype was similar to the range of the YYA0 diplotype, despite a significant difference in the median values $(P<0.0001)$.

$M B L 2$ expression in CF patients and parents. Nonparametric analysis of the median normalized MBL2 expression between CF patients and the parent (control) group demonstrated that CF patients had significantly higher levels of MBL2 protein (Figure 1C and Supplemental Table 3). The higher MBL2 expression in CF patients was most likely associated with induction of MBL2 due to the persistent bacterial challenge and mobilization of innate defense mechanisms. The lack of significant elevation in MBL2 expression in the ${ }^{* *} 00$ diplotypes almost certainly resulted from the structural mutations that prevent oligomerization of the protein which in turn leads to an inability to detect the MBL2 oligomer in the assay system.

$M B L 2$ expression in the pediatric and adult cohorts. To investigate a potential effect of age on MBL2 expression, the CF patients were divided into pediatric (age $<18.5 \mathrm{yr} ; n=848$ ) and adult (age $\geq 18.5$ yr; $n=545$ ) groups. The pediatric group had significantly higher plasma levels of MBL2 than the adult group for all diplotypes except $* 00$ (Figure 1D).

$M B L 2$ and age offirst $P$. aeruginosa infection. Figure 2 shows the KaplanMeier curves for the 3 MBL2 diplotypes in the pediatric cohort. The median age of first $P$. aeruginos a infection was significantly different for low, intermediate, and high $M B L 2$ groups $(4.4,7.0$, and $8.0 \mathrm{yr}$, respectively; $P=0.0003)$, indicating that MBL2 deficiency is associated with significantly earlier onset of $P$. aeruginosa infection.

Polymorphisms in the TGFB1 gene influence age of onset of first $P$. aeruginosa infection in MBL2-deficient CF patients. High-expressing variants of TGFB1 have been shown to be associated with a more severe lung disease phenotype (25). To investigate whether this could be explained by early $P$. aeruginosa infection, we analyzed age of first infection for the TGFB1 codon 10 (rs1982073) genotypes in the pediatric CF cohort. There was no difference in the age of first $P$. aeruginosa infection between the genotypes $(P=0.7$; Supplemental Figure 1$)$.

Analysis of the MBL2 diplotype groups stratified by the TGFB1 codon 10 genotypes demonstrated an influence of the high-expressing TGFB1 allele $C$ on age of first $P$. aeruginosa infection among the 3 $M B L 2$ diplotype groups. No effect of $M B L 2$ diplotype on age of first infection was observed in the subset of patients carrying the low-expressing TGFB1 genotype TT ( $P=0.16$; Figure $3 \mathrm{~A})$. However, in the subjects homozygous for the high-expressing TGFB1 C allele (CC genotype), the differences in median age of first $P$. aeruginosa infection by $M B L 2$ diplotype group were magnified (low $M B L 2,3.1 \mathrm{yr}$; intermediate $M B L 2$, $6.7 \mathrm{yr}$; high $M B L 2,8.7 \mathrm{yr} ; P=0.01$; Figure $3 \mathrm{C}$ ). In patients with low levels of MBL2, the CC genotype was associated with the earliest infection age, while the TT genotype was associated with the oldest age at first infection. The TGFB1 variant (rs2241715) exhibited a similar result as that observed for the codon 10 variant (data not shown). Although this observation suggested the possibility of gene-gene interaction between MBL2 and TGFB1, testing by the Cox proportional hazards regression model failed to detect such interaction ( $P=0.2$ for the interaction term $M B L 2 * T G F B 1)$.

MBL2 and MBL2/TGFB1 modulation of pulmonary function in CF patients. Of the patients studied, 511 were old enough to have multiple pulmonary function measurements in the 3 years before study enrollment. In a mixed regression model of forced expiratory volume in $1 \mathrm{~s}$ as a percentage of the predicted value $\left(\mathrm{FEV}_{1} \%\right.$ predicted; see Methods) versus age, MBL2, TGFB1, and all interaction terms, the 3-way interaction was significant $(P=0.002)$, indicating that the slope of $\mathrm{FEV}_{1}$ versus age was affected by MBL2 and TGFB1 genotypes. Table 5 shows how the interaction of these modifiers affected lung function. Rate of decline of $\mathrm{FEV}_{1}$ was significantly steeper in the $M B L 2$ diplotype group associated with low expression $(P=0.05)$. When stratified by TGFB1 genotype, the significant effect of MBL2 on $\mathrm{FEV}_{1}$ was only seen in the high-expressing CC genotype group $(P=0.0002)$. The intercept at age 10 was relatively stable across all genotype combinations, except in the high TGFB1/low MBL2 group, where it was significantly lower. Analysis of TGFB1 codon 10 alone showed a significant effect on lung function, consistent with earlier reports. The rate of $\mathrm{FEV}_{1}$ decline was $-2.62 \%$ ( $95 \% \mathrm{CI},-3.29 \%$ to $-1.95 \%),-1.22 \%$ (95\% CI, $-1.61 \%$ to $-0.83 \%)$, and $-2.04 \%$ (95\% CI, $-2.45 \%$ to $-1.62 \%)$ per year in CC, CT, and TT genotypes, respectively $(P=0.0005)$, while the respective intercepts were $88.5 \%, 87.5 \%$, and $86.3 \%(P=0.7)$.

Taken together, these data suggest that the effect of MBL2 expression is a critical determinant of the severity of lung disease at a young age in patients with $\mathrm{CF}$ disease and that high-expressing variants of TGFB1 enhance this effect. Age at first infection was a significant predictor of $\mathrm{FEV}_{1}$ intercept at age 10 (age $<6 \mathrm{yr}$,

\section{Table 3}

$M B L 2$ gene variants in this study: diplotypes

$\begin{array}{lcc}\text { Diplotype } & \begin{array}{c}\text { Predicted genotype } \\ \text { combinations }\end{array} & \begin{array}{c}\text { Simplified } \\ \text { notation } \\ \text { YO/YO + XO/YO }\end{array} \\ 1 & \text { XA/YO } & { }^{* *} 00^{B} \\ 2^{C} & \text { YA/YO } & \text { XYAO } \\ 3 & \text { XA/XA } & \text { YYAO } \\ 4 & \text { XA/YA } & \text { XXAA } \\ 5 & \text { YA/YA } & \text { XYAA } \\ 6 & Y Y A\end{array}$

AThe phase prediction of the B, C, and D structural mutations (see Table 2) with the $X Y$ variant in the promoter region (-221) were based on the very high linkage disequilibrium between these mutations and $Y$ allele of the $X Y$ variant. ${ }^{B}$ The extremely rare diplotype $X Y 00$ was combined with the YYOO genotype as the ${ }^{\star *} 00$ diplotype. CDiplotype 2 was inferred. 
Table 4

$M B L 2$ gene variants in this study: diplotype groups

\section{Diplotype combination}

YO/YO, XO/YO, and XA/YO

$Y A / Y O$ and $X A / X A$

$X A / Y A$ and $Y A / Y A$

\section{Diplotype group}

Low MBL2

Intermediate $M B L 2$

High MBL2
$82.2 \%, n=161$; age $\geq 6 \mathrm{yr}, 89.3 \%, n=350 ; P=0.0001)$ but not of rate of decline $(-1.95$ and -1.72 , respectively; $P=0.4)$. When infection age was added to the models of $\mathrm{FEV}_{1}$ versus $M B L 2$ or TGFB1, both infection age and genotype remained significant predictors of lung function (data not shown).

Effect of gender on age at first infection and lung function. The age at first $P$. aeruginosa infection was significantly lower in females than in males (median age, 6.7 versus 7.9 years; $P=0.04$, log- rank test). However there was no interaction between gender and MBL2 diplotype group $(P=0.62)$ or TGFB1 codon $10(P=0.98)$ in a Cox regression model.

Consistent results were seen for lung function. Females showed a more rapid mean rate of decline in $\mathrm{FEV}_{1}$ than males $(-1.45 \%$ versus $-1.08 \%$ predicted per year; $P<0.0001)$, but there was a similar intercept at age 10 years $(87.3 \%$ versus $86.7 \%$; $P=0.73$ ). Sex and the 3-way interaction of sex, age, and genotype were added to mixed models of $\mathrm{FEV}_{1}$ based on MBL2 or TGFB1, and there was no suggestion that the effects of either were different for males and females. The 3-way interaction terms were not significant in the MBL2 model $(P=0.80)$ or in the codon 10 model $(P=0.41)$. The effect of gender on the interaction between TGFB1 codon 10 and MBL2 diplotype groups for lung function decline could not be tested. This regression model involves a 4-way interaction and all possible 2- and 3-way interactions, and the number of patients in the smallest categories of sex, $M B L 2$ group, and TGFB1 group were insufficient.
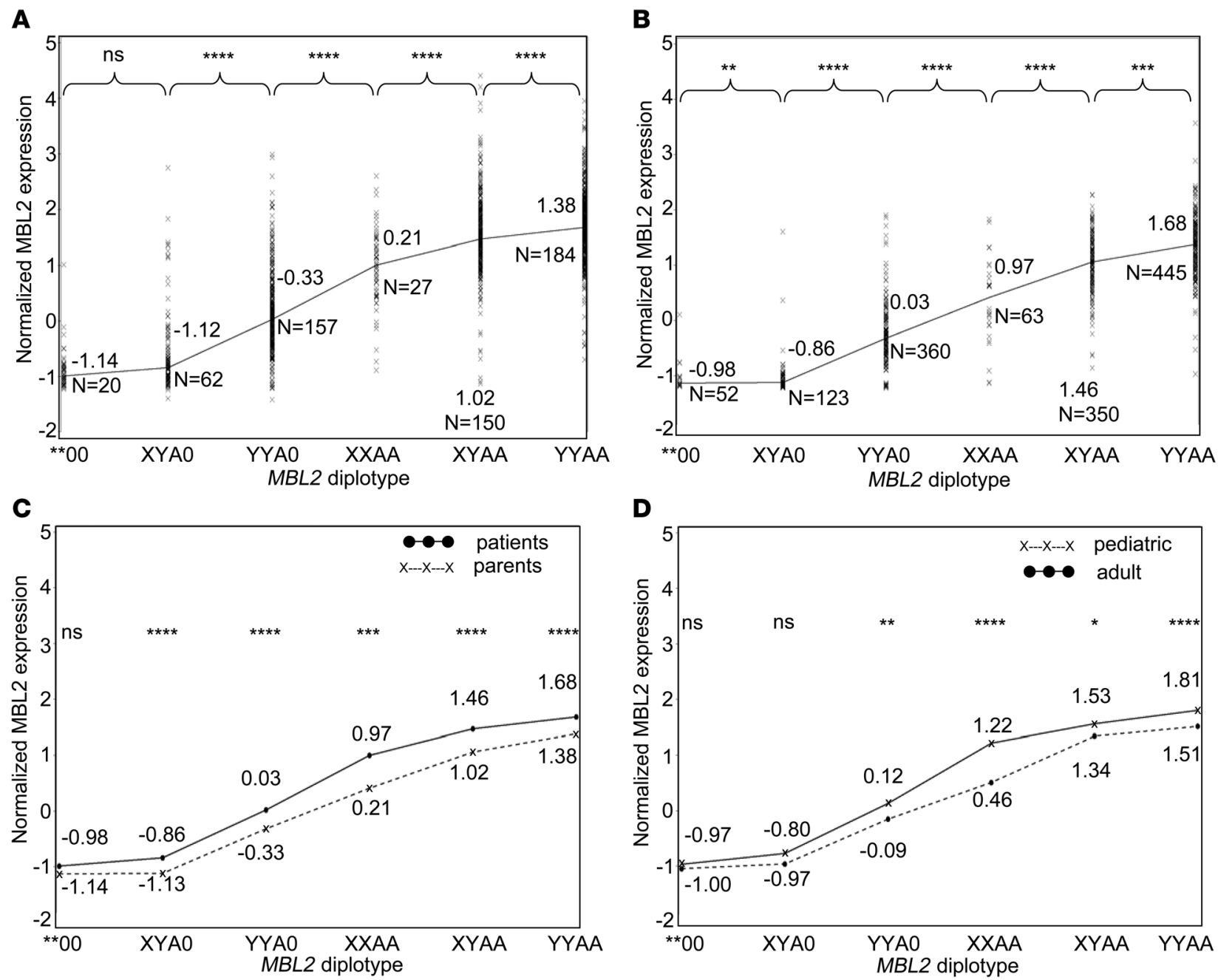

D

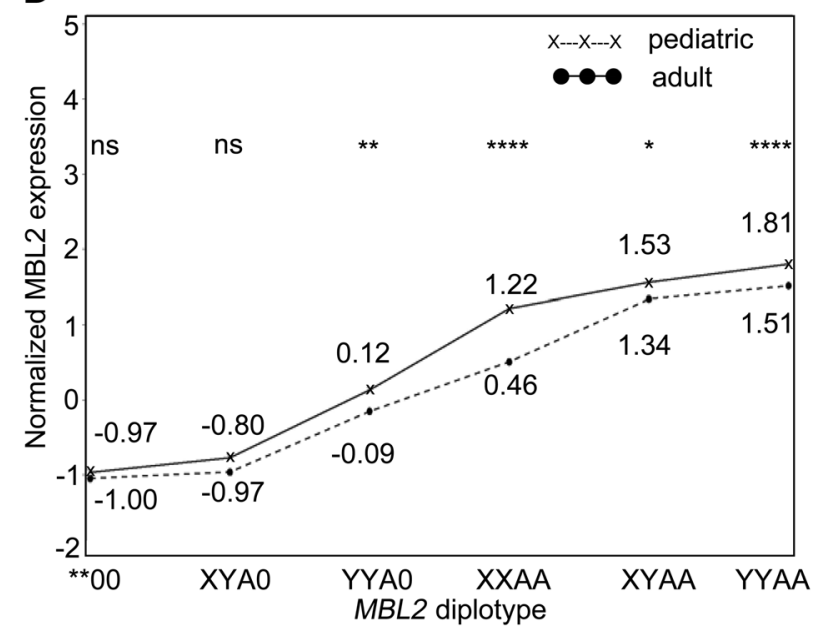

Figure 1

Normalized MBL2 levels corresponding to 6 MBL2 gene diplotypes $\left({ }^{\star *} 00, \mathrm{XYA0}, \mathrm{YYA0}, \mathrm{XXAA}, \mathrm{XYAA}\right.$, and YYAA) in CF patient and parent cohorts. (A and B) Distribution of normalized MBL2 expression values and median MBL2 expression values for each diplotype is shown for 600 parents (A) and 1,393 PI CF patients (B). (C and $\mathbf{D})$ Comparison of normalized MBL2 expression values between patients and parents (C) and between 848 pediatric and 545 adult patients (D). Statistical difference was tested using the Wilcoxon rank-sum test. ${ }^{\star} P<0.05$; ${ }^{* *} P<0.01 ;{ }^{* *} P<0.001 ;{ }^{* * *} P<0.0001$. 


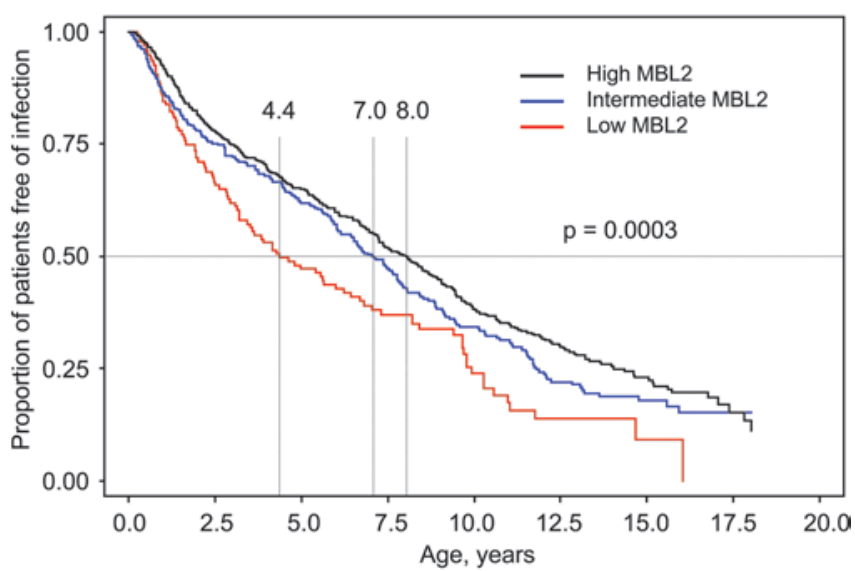

MBL2 and age at first infection in $\triangle F 508$ homozygotes. Table 1 demonstrates that lung function and age at first infection were similar in patients who were homozygous for the $\Delta \mathrm{F} 508$ mutation and other PI

\section{Figure 2}

Results of time-to-event analysis showing median age and $95 \% \mathrm{Cl}$ at first positive culture for $P$. aeruginosa. The proportion of patients not infected with $P$. aeruginosa versus age is plotted for low $M B L 2$ ( ${ }^{* *} 00$ and XYA0; $n=136$ ), intermediate MBL2 (YYA0 and XXAA; $n=311$ ), and high MBL2 (YYAA and XYAA; $n=572$ ) diplotype groups. The graph shows data for 1,019 pediatric $(<18.5$ years of age) PI CF patients. Median age of infection was $4.4 \mathrm{yr}(95 \% \mathrm{Cl}$, 3.2 to $6.4 \mathrm{yr}$ ) for the low MBL2 group, $7.0 \mathrm{yr}(95 \% \mathrm{Cl}, 6.1$ to $7.9 \mathrm{yr})$ for the intermediate $M B L 2$ group, and $8.0 \mathrm{yr}(95 \% \mathrm{Cl}, 7.2$ to $8.8 \mathrm{yr})$ for the high $M B L 2$ group. The survival probability differences were tested using log-rank test $(P=0.0003)$.

patients. The effect of MBL2 on age at first infection was computed for this subset homozygous for $\Delta \mathrm{F} 508$, and the results were consistent with the main analysis. The median age at first infection was $3.9 \mathrm{yr}$ (95\% CI, 2.9 to $9.7 \mathrm{yr}$ ), $7.4 \mathrm{yr}$ (95\% CI, 6.1 to $8.4 \mathrm{yr}$ ), and $8.1 \mathrm{yr}$ (95\% CI, 7.2 to $9.2 \mathrm{yr}$ ) for low, intermediate and high $M B L 2$ groups, respectively $(P=0.01, \log$-rank test). No further analysis was conducted on the subset of $\Delta \mathrm{F} 508$ homozygotes because of the small subgroup sizes.
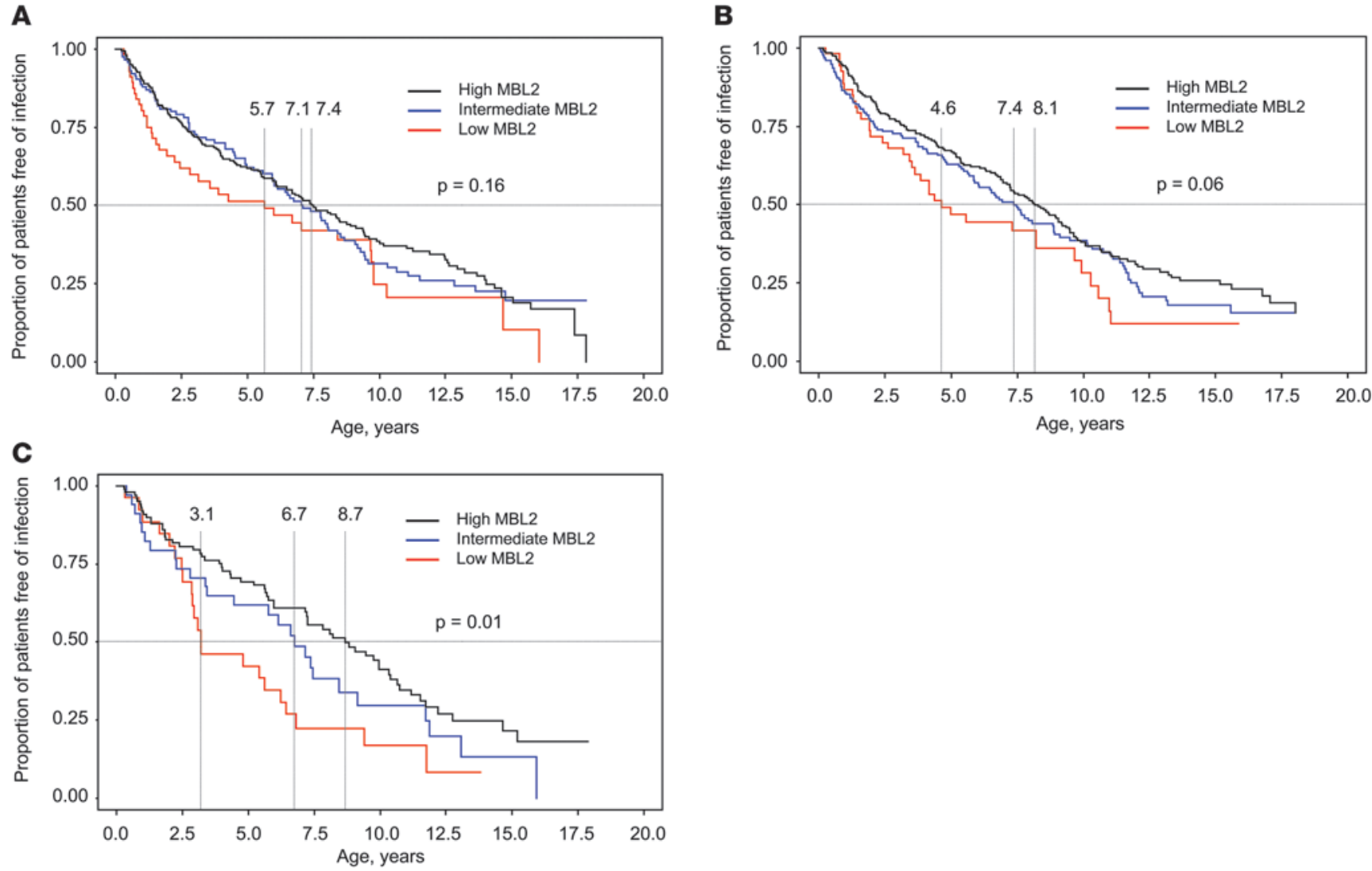

Figure 3

Results of time-to-event analysis (as in Figure 2) plotted for the 3 MBL2 diplotype groups and stratified by the codon 10 (rs1982073, L10P) TGFB1 genotypes. Data are shown for 1,003 pediatric PI CF patients; no codon 10 results were available for 16 patients. (A) For the TT genotype ( $n=389)$, median age at first positive $P$. aeruginosa culture was $5.7 \mathrm{yr}(95 \% \mathrm{Cl}, 2.4$ to $9.7 \mathrm{yr}$ ) for the low $\mathrm{MBL} 2 \mathrm{group}(n=56), 7.1 \mathrm{yr}(95 \% \mathrm{Cl}$, 5.9 to $8.5 \mathrm{yr})$ for the intermediate MBL2 group $(n=115)$, and $7.4 \mathrm{yr}(95 \% \mathrm{Cl}, 6.1$ to $9.1 \mathrm{yr})$ for the high $M B L 2$ group $(n=218)$. (B) For the CT genotype $(n=454)$, median age at first positive $P$. aeruginosa culture was $4.6 \mathrm{yr}(95 \% \mathrm{Cl}, 3.5$ to $9.7 \mathrm{yr})$ for the low $M B L 2 \mathrm{group}(n=53), 7.4 \mathrm{yr}$ $(95 \% \mathrm{Cl}, 5.9$ to $8.9 \mathrm{yr})$ for the intermediate $\mathrm{MBL} 2$ group $(n=155)$, and $8.1 \mathrm{yr}(95 \% \mathrm{Cl}, 7.1$ to $9.4 \mathrm{yr})$ for the high $M B L 2 \mathrm{group}(n=246)$. (C) For the CC genotype $(n=160)$, median age at first positive $P$. aeruginosa culture was $3.1 \mathrm{yr}(95 \% \mathrm{Cl}, 2.8$ to $6.2 \mathrm{yr})$ for the low MBL2 group ( $n=26)$, $6.7 \mathrm{yr}(95 \% \mathrm{Cl}, 3.4$ to $9.1 \mathrm{yr})$ for the intermediate $\mathrm{MBL} 2$ group $(n=34)$, and $8.7 \mathrm{yr}(95 \% \mathrm{Cl}, 7.1$ to $10.4 \mathrm{yr})$ for the high $\mathrm{MBL} 2 \mathrm{group}(n=100)$. Differences between groups were determined by the log-rank test. 


\section{Table 5}

Mixed-model analyses of $\mathrm{FEV}_{1} \%$ predicted versus age, MBL2 diplotype group, and codon 10 TGFB1 genotype in pediatric CF patients (<18.5 yr)

\begin{tabular}{|c|c|c|c|c|}
\hline & Low MBL2 group & Intermediate $M B L 2$ group & High MBL2 group & $P$ \\
\hline \multicolumn{5}{|c|}{ All patients ${ }^{A}(n=511)$} \\
\hline $\mathrm{FEV}_{1} \%, 10 \mathrm{yr}$ & 85.1 & 87.4 & 87.2 & 0.70 \\
\hline Slope (95\% Cl) & $-2.57(-3.3$ to -1.8$)$ & $-1.83(-2.3$ to -1.4$)$ & $-1.56(-1.9$ to -1.2$)$ & 0.05 \\
\hline$n$ & 62 & 160 & 289 & \\
\hline \multicolumn{5}{|c|}{ Codon 10 genotype TT ( $n=197)$} \\
\hline $\mathrm{FEV}_{1} \%, 10 \mathrm{yr}$ & 83.9 & 86.7 & 86.2 & 0.80 \\
\hline Slope $(95 \% \mathrm{Cl})$ & $-2.35(-3.4$ to -1.3$)$ & $-1.56(-2.3$ to -0.8$)$ & $-2.16(-2.7$ to -1.6$)$ & 0.36 \\
\hline$n$ & 30 & 61 & 106 & \\
\hline \multicolumn{5}{|c|}{ Codon 10 genotype CT ( $n=233$ ) } \\
\hline $\mathrm{FEV}_{1} \%, 10 \mathrm{yr}$ & 87.2 & 85.8 & 88.5 & 0.65 \\
\hline Slope $(95 \% \mathrm{CI})$ & $-1.00(-2.4$ to 0.4$)$ & $-1.53(-2.2$ to -0.9$)$ & $-1.09(-1.6$ to -0.6$)$ & 0.56 \\
\hline$n$ & 21 & 78 & 134 & \\
\hline \multicolumn{5}{|c|}{ Codon 10 genotype CC ( $n=81)$} \\
\hline $\mathrm{FEV}_{1} \%, 10 \mathrm{yr}$ & 81.3 & 95.1 & 86.4 & 0.11 \\
\hline Slope $(95 \% \mathrm{Cl})$ & $-5.52(-7.3$ to -3.7$)$ & $-3.47(-4.8$ to -2.2$)$ & $-1.55(-2.4$ to -0.7$)$ & 0.0002 \\
\hline$n$ & 11 & 21 & 49 & \\
\hline
\end{tabular}

Shown are mixed-model estimates of mean $\mathrm{FEV}_{1} \%$ predicted at 10 years of age and mean slope of $\mathrm{FEV}$ \% predicted versus age. In a model based on age, TGFB1 codon 10 genotype, and MBL2 genotype group, there was a 3-way interaction $(P=0.002)$ of slope with $M B L 2$ and $T G F B 1$. Therefore, separate models of $\mathrm{FEV}_{1}$ versus age and MBL2 were computed for each TGFB1 codon 10 genotype. APatients with at least 2 measurements of FEV ${ }_{1}$ between 6 and 18 years of age.

\section{Discussion}

Lung disease, the major life-limiting complication of CF, is poorly correlated with mutations in the disease-causing gene, CFTR (2). Emerging data from various studies of CF suggest a multifactorial modulation of lung disease severity, including contributions of genetic, environmental, and stochastic factors (1). Studies of heritability of lung disease severity in CF demonstrated a substantial contribution of secondary genetic factors $(38,39)$. The estimated heritability of 0.68 is very high and indicates strong impact of secondary genetic component in controlling variation in CF lung disease severity (39). Although the number of pulmonary CF modifiers is unknown, the relative contribution of several genes can be inferred from current studies (25). For example, TGF- $\beta 1$ is responsible for only $15 \%-20 \%$ of the variability in CF lung disease severity, suggesting involvement of additional modifiers $(25,40)$. The complex inheritance of lung disease severity in CF may involve not only the contribution of multiple genes, but also interactions between them.

Our study thus offers insight into the complex genetics of CF modulation by demonstrating, for the first time to our knowledge, comodulation of traits related to CF lung disease by 2 genes, $M B L 2$ and TGFB1, as well as providing evidence for their interaction in controlling clinical variation of pulmonary phenotype in pediatric CF patients. We demonstrated that MBL2 deficiency was associated with a significant increase in the risk of early infection with $P$. aeruginosa and rate of decline of pulmonary function in pediatric CF patients. While polymorphisms in TGFB1 alone did not have an impact on age of acquisition of $P$. aeruginosa, we provided evidence that they significantly influenced its modulation by MBL2 and that the 2 genes interact to influence pulmonary function. Our results may explain apparently contradictory findings in previous reports, but replication of the combined gene effect would require a larger number of young patients than is available at present. Close follow-up of young patients in this and other cohorts will be necessary to confirm and refine the complex association.
The patient cohort was recruited from a population-based sample representing nearly $75 \%$ of the current Canadian CF population (34). The resource consists of DNA and plasma samples from CF patients and parents as well as demographic and clinical data from patients. Comparison with the Canadian CF Patient Registry demonstrates that the demographic and clinical data in the recruited patient cohort were nationally representative (34). Consequently, the results obtained from these cohorts can be generalized to the entire Canadian CF population. Treatment and survival of CF patients has been steadily improving over the 2 decades in which the study patients were born. All analyses in this report were repeated, including a binary birth cohort variable. In no case did birth cohort affect the results of MBL2 and TGFB1 genotypes (data not shown).

Secondary modulation of the severity of CF lung disease can be confounded by CFTR genotype, the primary determinant of disease severity. Patients carrying at least 1 mild CFTR mutation tend to have a milder form of CF (i.e., almost exclusively pancreatic sufficiency). Pulmonary disease is somewhat milder, although highly variable, than in patients carrying severe mutations on both alleles (due to lack of CFTR protein synthesis, processing, or function). Therefore, to avoid a potential confounding effect of CFTR genotypes, only pancreatic insufficient patients who had verified severe CFTR mutations on both alleles were included in this study. The demographic and clinical characteristics of the study population (Table 1) demonstrate that the subset of patients homozygous for the $\Delta$ F508 mutation were remarkably similar to the cohort patients carrying other severe CFTR genotypes, justifying the combination of the 2 patient groups as the study population for our analyses.

To our knowledge, this is the largest and most comprehensive study of MBL2 as a CF pulmonary disease modifier. We verified previous findings on the relationship between genotypes of the key $M B L 2$ variants and expression of the MBL2 protein in peripheral blood $(4,12,19)$. We also classified $M B L 2$ diplotypes on the basis of plasma protein expression of oligomerized MBL2 and genotyped for the variants affecting production or assembly of 
MBL2 protein (Table 2). Our results (Figure 1, A and B) confirmed earlier reports $(4,12,19)$ showing a correlation between the 6 $M B L 2$ diplotypes and corresponding MBL2 expression levels. It should be noted, however, that there was considerable variation of MBL2 expression between subjects in each diplotype cohort. Other unspecified factors, such as unrecognized $M B L 2$ mutations and modulation of MBL2 expression by disease factors, could account for the variable MBL2 expression levels (41).

Carlsson et al. (23) reported an increased plasma level of MBL2 in CF patients. The results of the present study not only confirmed this observation, but also demonstrated that pediatric CF patients had significantly higher MBL2 plasma levels than adults. The higher MBL2 expression in pediatric CF patients could be attributed to an enhanced acute inflammatory response due to repeated bacterial challenge, which is not observed in older patients who have long-established chronic sinopulmonary infections.

In a previous study, Garred et al. (4) demonstrated an earlier age of onset of chronic $P$. aeruginosa infection in CF patients as a result of MBL2 deficiency. Because it is difficult to reliably establish the onset of chronic $P$. aeruginosa infection, we used age at the first positive respiratory culture as a surrogate. The time-to-event analysis showed that MBL2 deficiency was strongly associated with earlier acquisition of $P$. aeruginosa in young children with CF $(P=0.0003$; Figure 2$)$. This modulatory effect most likely reflects the critical role of MBL2 in the first defense against bacterial colonization (16) and supports the similar but less significant effect previously observed in a small Italian CF patient cohort (42). However, it should be also noted that although MBL2 deficiency has been implicated in many studies as contributing to higher susceptibility to infections, there are also data suggesting the opposite (43). This MBL duality may account for the relatively high frequency of MBL variants encoding low MBL serum concentrations in some populations and should be seen in the context of specific circumstances of bacterial challenge and infection (43).

Notably, the MBL2-deficient patients carrying the high-expressing TGFB1 genotype CC had the most significant earlier onset of $P$. aeruginosa acquisition. Interestingly, although TGFB1 affected the modulation of age of onset by MBL2 in the pediatric patients, no direct impact of its codon 10 genotypes was observed in the time-to-event analysis using the same cohort unstratified for MBL2 (Supplemental Figure 1). This modulation of the MBL2 effect by TGF- $\beta 1$ could result from impaired bacterial eradication and clearance in airways due to macrophage inactivation by elevated levels of TGF- $\beta 1(44,45)$. This, in conjunction with the MBL2 deficiency, would lead to the enhanced vulnerability of young CF patients to infection, manifesting as significantly earlier $P$. aeruginosa acquisition. This is in agreement with the well-documented dual role of TGF- $\beta 1$ as a pro- and antiinflammatory cytokine in the immune defense system $(46,47)$.

Because CF children with MBL2 deficiency acquire $P$. aeruginosa pulmonary infections at a significantly younger age than those with normal levels, one might expect that MBL2-deficient children would have faster decline of pulmonary function. Indeed, as shown in Table 5, we demonstrated that MBL2 deficiency correlated with a more rapid rate of decline of pulmonary function. The observed effect could be explained by the recognized role of MBL2 in immunity. Because MBL2 deficiency prevents the activation of the MBL2associated complement pathway, there is failure of the primary antibacterial defense, namely macrophage phagocytosis of bacteria. In this regard, MBL2 is not only involved in innate antibacterial defense through the complement pathway, it is also a potent regulator of inflammatory pathways (9), which are highly dependent on the concentration of circulating MBL2. Low plasma MBL2 levels induce monocyte production of proinflammatory cytokines, whereas high MBL2 levels have a strong antiinflammatory effect (9). This observation provides a potential explanation for the magnified modulatory effect on the severity of lung disease in the MBL2-deficient children homozygous for the high-producing TGFB1 codon 10 variant $C$ $(P=0.0002)$. Specifically, MBL2 deficiency $(<4 \mu \mathrm{g} / \mathrm{ml})$ has been shown to stimulate release of proinflammatory cytokines (TNF- $\alpha$, IL-1B, and IL-6) from monocytes, whereas cytokine production is greatly suppressed with MBL2 levels higher than $6 \mu \mathrm{g} / \mathrm{ml}$ (9). The context of MBL2-induced high levels of proinflammatory cytokines could support activation of the TGF- $\beta 1$-stimulated commitment of Th17 (i.e., IL-17-producing CD4 ${ }^{+}$) T cell lineage producing neutrophil-mobilizing cytokines instrumental in the host defense to infections (46-49). In this situation, enhanced production of TGF- $\beta 1$ by the $\mathrm{C}$ allele could further inhibit the host defense by a variety of mechanisms. These include downregulation of macrophage receptors critical for phagocytosis $(44,50)$, downregulation of $\operatorname{IgG}$ receptors on monocytic cells, and reduction of phagocytosis of IgGopsonized bacteria (51) as well as other mechanisms critical for antibacterial defenses, such as attenuation of innate immune signaling pathways mediated by TLR4 on LPS-induced macrophages (52).

The 2 clinical variables used in this analysis, namely the onset of lung infection and rate of decline of pulmonary function, have different contributions to CF lung pathophysiology. The first is a causative event, because lung infection is presumed to be an initiating event leading to progressive lung damage. The second is an outcome measure. Although the rate of decline of pulmonary function is clearly influenced by infection status, many other environmental and genetic factors will have protective or deleterious consequences. In this regard, statistical modeling of gene-gene interactions between $M B L 2$ and TGFB1 revealed strong interaction for lung function $(P<0.002)$ but not for the age of first $P$. aeruginosa infection $(P=0.2)$. Failure to detect $M B L 2 / T G F B 1$ interaction for the infection variable may reflect the imprecision in assessment of first $P$. aeruginosa infection. Nevertheless, these results warrant investigation of the biochemical and physiological mechanisms of interaction between these 2 proteins.

Consideration should be given to the clinical implications of these findings. Genetic testing for MBL2 and TGFB1 variants may permit early identification of at-risk patients either at diagnosis or through newborn screening. This would allow physicians to closely monitor these subjects and give consideration to more aggressive efforts to prevent early onset of bacterial infections. Knowledge of specific modifier genotypes would also permit more accurate patient stratification for entry into therapeutic clinical trials. However, incorporation of genotyping into clinical trials and practice awaits determination of sensitivity and specificity for Pseudomonas prediction, ideally in a population of patients identified by newborn screening. Finally, recognition of the clinical consequences of MBL2 deficiency may have therapeutic implications. For example, intravenous administration of human MBL2 derived from pooled plasma to an MBL2-deficient CF patient with end-stage lung disease was considered to have some beneficial effects (53). Nevertheless, our findings suggest that success with MBL2 replacement therapy would require that it be administered prior to the onset of chronic bacterial infection. Now that recombinant MBL2 protein is available, there is wide interest in MBL2 supplementation. Trials are in progress 
with recombinant MBL2 protein therapy in MBL2-deficient adults who are at increased risk of infection during chemotherapy or following liver or bone marrow transplantation $(54,55)$. A therapeutic trial with a recombinant MBL2 protein has also been proposed for MBL2-deficient CF patients (56). More research has to be done to refine the details of MBL2 modulation before seriously considering clinical trials of human recombinant MBL2 replacement therapy to delay or prevent early onset of chronic pulmonary infections in CF disease. However, MBL2-deficient patients may benefit from more frequent microbiological screening in order to detect earlier signs of infection that may respond to current antibiotic treatment.

In conclusion, based on its critical role in innate antibacterial defense, we postulate that MBL2 contributes to the variability of lung disease by modulating the age of acquisition of $P$. aeruginos $a$ in pediatric CF patients. Furthermore, we provide evidence of a complex interaction between MBL2 and TGF- $\beta 1$ and rate of decline of pulmonary function in young patients with $\mathrm{CF}$, possibly related to a synergistic effect on the onset of acquisition of $P$. aeruginosa infection. Gaining insight into the mechanisms of MBL2 modulation of lung pathophysiology in CF patients and its interaction with other pulmonary modifiers (e.g., TGF- $\beta 1$ ) will be critical to explain our findings at a molecular level and provide a basis for developing modifier-based therapies.

\section{Methods}

CF patients and parent cohorts. CF patients and their family members were recruited from 37 specialized CF clinics across Canada (shown in Supplemental Table 5), during a period from 2003 to 2006, as participants in the Canadian CF Modifier Study. The protocols for this study were approved by ethical review boards at the Hospital for Sick Children and all participating institutions. Informed consent was obtained from each individual or his/her guardian. The Canadian CF Modifier Study population consists of 2,441 patients diagnosed with CF between 1951 and 2006 and 3,092 parents. This report of first infection as a phenotype was restricted to pediatric patients ( $<18.5$ years of age at the time of recruitment) in order to capture the most reliable information on first infection and to compute average lung function decline unbiased by mortality selection. The age of first infection was generally not available in the records of patients reported from clinics specializing in adult CF care. CF patients included in this study had PI and carried severe CFTR mutations on both alleles. Patients reported as PI but carrying at least 1 CFTR mutation typically associated with pancreatic sufficiency were excluded. The demographic and clinical characteristics of the pediatric patient cohort are shown in Table 1.

Pulmonary phenotypes. To evaluate MBL2 (and MBL2 stratified by TGF- $\beta 1$ variants) as a pulmonary CF modifier, 2 lung-related clinical outcome variables were used: age at first $P$. aeruginosa infection and $\mathrm{FEV}_{1}$. Patients attending Canadian CF clinics have sputum cultures and lung function testing at every clinic visit, which are routinely scheduled at 3-month intervals. Age at the time of the first positive respiratory culture for P. aeruginosa was used as an indicator of susceptibility to infection with this bacterium. $\mathrm{FEV}_{1}$ (as percent of predicted for sex, height, and age) reflects the progression of CF lung disease and is the best predictor of survival (57). Predicted values were based on the formula of Knudson et al. (58) for those over the age of 10 years and Corey et al. (59) for those 6-10 years of age. FEV 1 measurements in the 3 years prior to enrollment were recorded in order to capture the current status and rate of decline for each patient.

Plasma and DNA samples. Individual blood samples were collected in glass tubes containing anticoagulant (Acid Citrate Dextrose, ACD-Vacutainer tubes; $\mathrm{BD}$ ) and maintained at room temperature. Plasma samples were isolated by centrifugation at $524 \mathrm{~g}$ for 10 minutes and immediately frozen and stored at $-70^{\circ} \mathrm{C}$. Genomic DNA was extracted using the phenol/chloroform procedure (60). The DNA stocks were stored at $4^{\circ} \mathrm{C}$. Patients were recruited during the years 2003-2006, and the age in all analyses is based on the date the blood sample was taken.

Genotyping. For MBL2, 4 functional MBL2 gene variants were genotyped in genomic DNA samples: the promoter variant at position -221 (Y or X variant) and 3 structural mutations in exon 1 (B, C, and D variants), as shown in Table 2. The genotyping was performed using Luminex Bead Array technology $(61,62)$ implemented for the $M B L 2$ variants as described in Supplemental Methods and Supplemental Table 1. MBL2 diplotypes (Table 3) were inferred from promoter variants $\mathrm{X}$ and $\mathrm{Y}$ and exon 1 structural variants A, B, C, and D according to Madsen et al. (11).

For TGFB1, the codon 10 expression variant (rs1982073, L10P) in TGFB1 was genotyped using TaqMan genotyping assay (63). Genotyping of the promoter variant (-509; rs1800469) was performed using Luminex Bead Array technology (Luminex Corp.).

Measurement of MBL2 protein plasma levels. The plasma level of oligomerized MBL2 protein was measured in blood samples from 1,393 CF patients and 600 parents as described in Supplemental Methods. The MBL2 protein levels were normalized to adjust for batch differences using the mean and standard deviation for 10 non-CF control samples included in every batch. Therefore the normalized MBL2 expression level reflects the variation above or below that expected for samples unrelated to CF.

Statistics. Patients who had not acquired $P$. aeruginosa infection at the time of observation will likely acquire the infection in the future; therefore, their age at first infection is unknown. To account for this, age at first infection was treated as a right-censored variable, and time-to-event analysis was used to compare age at first infection in different subgroups. Because infection is present for some unknown time before being cultured, the age at first positive culture is a left-censored estimate of true infection age. Left censoring is not considered in standard time-to-event analysis. Restriction of the study subjects to children and to those followed in specialized CF clinics minimizes the unknown time from infection to detection. We plotted Kaplan-Meier survival curves to demonstrate the distribution of age at first $P$. aeruginosa infection, and curves were compared using the log-rank statistic. P. aeruginosa-free survival curves were compared for $M B L 2$ genotype groups, for TGFB1 genotypes, and for MBL2/TGFB1 combinations. Cox proportional hazards regression was used to test the effects of sex and of multiple genotype groups on age at first infection.

Pulmonary function testing involves volitional breathing maneuvers that are generally not possible in children until 5-7 years of age. Data on current lung function and decline over the past 3 years, represented by $\mathrm{FEV}_{1}$ $\%$ predicted, were compiled for patients that had been tested. Longitudinal pulmonary function patterns (in patients with at least 2 measurements) were analyzed using a random effects mixed-model regression, as described by Schluchter et al. (64), and the mean intercept at 10 years of age and the mean slope were compared in potential modifier categories. The dependent variable in all models was $\mathrm{FEV}_{1} \%$ predicted, and the time variable was age $10 \mathrm{yr}$. Genotype groups for potential modifiers were included as categorical variables. Interaction terms for modifier categories and age provided a statistical test for category differences in the change of lung function over time. The effects of gender and age at first infection were also added to selected models. Because age at first infection was censored, it was dichotomized into $<6$ years and $\geq 6$ years, the latter including those not yet infected. All statistical analyses were performed using SAS version 9.1 software. A $P$ value of 0.05 or less was considered significant.

\section{Acknowledgments}

This project was supported by Genome Canada through the Ontario Genomics Institute per research agreement 2004- 
OGI-3-05, by the Canadian Cystic Fibrosis Foundation, by the Ontario Research Fund - Research Excellence Program, and by donations from the Lloyd Carr-Harris Foundation. R. Dorfman is supported by the joint Fellowship of Canadian Institutes of Health Research and Ontario Women's Health Council. The authors thank Nicole Anderson, Jennifer Breaton, Mary Cristofi, Roxanne Rousseau, and Michael Van Spall for their exceptional effort in recruiting and ascertaining Canadian CF families for the study as well as Gouri Mukeerje, Aditi Master, Fan Lin, Danuta Markiewicz, Qiuju Huang, Xiao-Wei Yuan, Dorota Stefanowicz, and Anluan O'Brien for their excellent technical assistance. The authors express gratitude to all CF patients and their families for participating in this study. The authors are indebted to the following individuals from member institutions of the Canadian Consortium for CF Genetic Studies (see Supplemental Table 5) for ascertaining patient data and blood samples from CF patients and their families: S. Aaron, P. Barrett, B. Beaurivage, Y. Berthiaume, P. Bigonesse, M. Boland, L. Boucher, J. Boucher, S. Bourgh, F. Brosseau, N.E. Brown, C. Brunoro, N. Bureau, A. Cantin, L. Charette, G. Cote, A. Dale, G. Davidson, K. Devesceri, R. Dicaire, V. Fauvel, A. Freitag, D.N. Garey, M. Gaul, W. Gervais, J. Gjevre, F. Gosse, A. Gravelle, B. Habbick, R. Hennessey, S.B. Holmes, J. Hopkins, D. Hughes, M. Jackson, J. Jacob, A. Jeanneret, P. Kean, W. Kepron, T. Kovesi, V.J. Kumar, L. Lands,
M. LaPerriere, J. Leong, R. Levesque, D. Lougheed, M. Lowe, B. Lyttle, K. Malhotra, J.E. Marcotte, S. Marsolais, C. Martineau, E. Matouk, D. McCulloch, R.T. Michael, M. Montgomery, R. Morris, E.M. Nakielna, F. Paquet, H. Pasterkamp, N. Patterson, L. Pedder, L. Peterson, N. Petit, C. Piche, M. Plante, H.R. Rabin, K. Ramlall, F. Raymong, L. Rivard, G. Rivard, M. Roussin, M. Ruel, J. Salgado, L. Semple, E. Sheppard, F. Simard, A. Smith, M. Solomon, R. Stackhouse, J. Tabak, L. Taylor, A. Tsang, E. Tullis, C. Turtle, K. Vandamheen, M. van Spall, R. van Wylick, I. Waters, T. Wells, S. Wiltse, and P. Zuberbuhler.

Received for publication August 28, 2007, and accepted in revised form January 2, 2008.

Address correspondence to: Julian Zielenski, Program in Genetics and Genome Biology, Hospital for Sick Children, 555 University Avenue, Toronto, Ontario M5G 1X8, Canada. Phone: (416) 813-5064, Fax: (416) 813-8856, E-mail: jziel@genet.sickkids.on.ca.

Lilian Pereira's present address is: Universidade Federal do Parana, Curitiba, Brazil.

Lap-Chee Tsui's present address is: University of Hong Kong, Pokfulam, People's Republic of China.
1. Cutting, G.R. 2005. Modifier genetics: cystic fibrosis. Annu. Rev. Genomics Hum. Genet. 6:237-260.

2. Dorfman, R., and Zielenski, J. 2006. Genotype-phenotype correlations in cystic fibrosis. In Cystic fibrosis in the 21 st century. A. Bush, E.W.F.W. Alton, J.C. Davies, U. Griesenbach, and A. Jaffe, editors. Karger Publishers. Basel, Switzerland. 61-68.

3. Knowles, M.R. 2006. Gene modifiers of lung disease. Curr. Opin. Pulm. Med. 12:416-421.

4. Garred, P., et al. 1999. Association of mannosebinding lectin gene heterogeneity with severity of lung disease and survival in cystic fibrosis. J. Clin. Invest. 104:431-437.

5. Dommett, R.M., Klein, N., and Turner, M.W. 2006. Mannose-binding lectin in innate immunity: past, present and future. Tissue Antigens. 68:193-209.

6. Worthley, D.L., Bardy, P.G., and Mullighan, C.G. 2005. Mannose-binding lectin: biology and clinical implications. Intern. Med. J. 35:548-555.

7. Fujita, T., Matsushita, M., and Endo, Y. 2004. The lectin-complement pathway - its role in innate immunity and evolution. Immunol. Rev. 198:185-202.

8. Fraser, D.A., et al. 2006. C1q and MBL, components of the innate immune system, influence monocyte cytokine expression. J. Leukoc. Biol. 80:107-116.

9. Jack, D.L., et al. 2001. Mannose-binding lectin regulates the inflammatory response of human professional phagocytes to Neisseria meningitidis serogroup B. J. Infect. Dis. 184:1152-1162.

10. Ratjen, F. 2006. What's new in CF airway inflammation: an update. Paediatr. Respir. Rev. 7(Suppl. 1):S70-S72.

11. Madsen, H.O., et al. 1995. Interplay between promoter and structural gene variants control basal serum level of mannan-binding protein. J. Immunol. 155:3013-3020.

12. Garred, P., Larsen, F., Madsen, H.O., and Koch, C. 2003. Mannose-binding lectin deficiency - revisited. Mol. Immunol. 40:73-84.

13. Larsen, F., Madsen, H.O., Sim, R.B., Koch, C., and Garred, P. 2004. Disease-associated mutations in human mannose-binding lectin compromise oligomerization and activity of the final protein. J. Biol. Chem. 279:21302-21311.

14. Terai, I., et al. 2003. Relationship between gene polymorphisms of mannose-binding lectin (MBL) and two molecular forms of MBL. Eur. J. Immunol. 33:2755-2763.

15. Kilpatrick, D.C. 2002. Mannan-binding lectin: clinical significance and applications. Biochim. Biophys. Acta. 1572:401-413.

16. Kilpatrick, D.C. 2002. Mannan-binding lectin and its role in innate immunity. Transfus. Med. 12:335-352.

17. Koch, A., et al. 2001. Acute respiratory tract infections and mannose-binding lectin insufficiency during early childhood. JAMA. 285:1316-1321.

18. Summerfield, J.A., Sumiya, M., Levin, M., and Turner, M.W. 1997. Association of mutations in mannose binding protein gene with childhood infection in consecutive hospital series. BMJ. 314:1229-1232.

19. Davies, J.C., Turner, M.W., and Klein, N. 2004. Impaired pulmonary status in cystic fibrosis adults with two mutated MBL-2 alleles. Eur. Respir. J. 24:798-804.

20. Muhlebach, M.S., et al. 2006. Association between mannan-binding lectin and impaired lung function in cystic fibrosis may be age-dependent. Clin. Exp. Immunol. 145:302-307.

21. Yarden, J., et al. 2004. Polymorphisms in the mannose binding lectin gene affect the cystic fibrosis pulmonary phenotype. J. Med. Genet. 41:629-633.

22. Buranawuti, K., et al. 2007. Variants in mannosebinding lectin and tumour necrosis factor alpha affect survival in cystic fibrosis. J. Med. Genet. 44:209-214.

23. Carlsson, M., et al. 2005. Deficiency of the mannanbinding lectin pathway of complement and poor outcome in cystic fibrosis: bacterial colonization may be decisive for a relationship. Clin. Exp. Immunol. 139:306-313.

24. Olesen, H.V., Jensenius, J.C., Steffensen, R., Thiel, S., and Schiotz, P.O. 2006. The mannan-binding lectin pathway and lung disease in cystic fibrosis--disfunction of mannan-binding lectin-associated serine protease 2 (MASP-2) may be a major modifier. Clin. Immunol. 121:324-331.

25. Drumm, M.L., et al. 2005. Genetic modifiers of lung disease in cystic fibrosis. N. Engl. J. Med. 353:1443-1453.
26. Fitzpatrick, D.R., and Bielefeldt-Ohmann, H. 1999. Transforming growth factor beta in infectious disease: always there for the host and the pathogen. Trends Microbiol. 7:232-236.

27. Lee, C.G., Kang, H.R., Homer, R.J., Chupp, G., and Elias, J.A. 2006. Transgenic modeling of transforming growth factor-beta(1): role of apoptosis in fibrosis and alveolar remodeling. Proc. Am. Thorac. Soc. 3:418-423.

28. Lucas, M., et al. 2006. Requirements for apoptotic cell contact in regulation of macrophage responses. J. Immunol. 177:4047-4054.

29. Shao, X., Rivera, J., Niang, R., Casadevall, A., and Goldman, D.L. 2005. A dual role for TGF-beta 1 in the control and persistence of fungal pneumonia. J. Immunol. 175:6757-6763.

30. Takabayshi, K., et al. 2006. Induction of a homeostatic circuit in lung tissue by microbial compounds. Immunity. 24:475-487.

31. Grainger, D.J., et al. 1999. Genetic control of the circulating concentration of transforming growth factor type beta1. Hum. Mol. Genet. 8:93-97.

32. Yamada, Y., et al. 1998. Association of a polymorphism of the transforming growth factor-beta1 gene with genetic susceptibility to osteoporosis in postmenopausal Japanese women. J. Bone Miner. Res. 13:1569-1576.

33. Arkwright, P.D., et al. 2000. TGF-beta(1) genotype and accelerated decline in lung function of patients with cystic fibrosis. Thorax. 55:459-462.

34. Taylor, C., et al. 2006. The Canadian CF modifier gene project: A nationally representative DNA and phenotype resource. Pediatr. Pulmonol. 29(Suppl.):362.

35. Tsui, L.C. 1992. Mutations and sequence variations detected in the cystic fibrosis transmembrane conductance regulator (CFTR) gene: a report from the Cystic Fibrosis Genetic Analysis Consortium. Hum. Mutat. 1:197-203.

36. Consortium, T.C.F.G.-P. 1993. Correlation between genotype and phenotype in patients with cystic fibrosis. The Cystic Fibrosis Genotype-Phenotype Consortium. N. Engl. J. Med. 329:1308-1313.

37. Kerem, E., et al. 1990. The relation between genotype and phenotype in cystic fibrosis - analysis of the most common mutation (delta F508). N. Engl. 
J. Med. 323:1517-1522.

38. Mekus, F., et al. 2000. Categories of deltaF508 homozygous cystic fibrosis twin and sibling pairs with distinct phenotypic characteristics. Twin Res. 3:277-293.

39. Vanscoy, L.L., et al. 2007. Heritability of lung disease severity in cystic fibrosis. Am.J. Respir. Crit. Care Med. 175:1036-1043.

40. Haston, C.K., and Hudson, T.J. 2005. Finding genetic modifiers of cystic fibrosis. N. Engl. J. Med. 353:1509-1511.

41. Bernig, T., et al. 2005. An analysis of genetic variation across the MBL2 locus in Dutch Caucasians indicates that 3 ' haplotypes could modify circulating levels of mannose-binding lectin. Hum. Genet. 118:404-415.

42. Trevisiol, C., et al. 2005. MBL2 polymorphisms screening in a regional Italian CF Center. J. Cyst. Fibros. 4:189-191.

43. Garred, P., Larsen, F., Seyfarth, J., Fujita, R., and Madsen, H.O. 2006. Mannose-binding lectin and its genetic variants. Genes Immun. 7:85-94.

44. Bottalico, L.A., Wager, R.E., Agellon, L.B., Assoian, R.K., and Tabas, I. 1991. Transforming growth factor-beta 1 inhibits scavenger receptor activity in THP-1 human macrophages. J. Biol. Chem. 266:22866-22871.

45. Tsunawaki, S., Sporn, M., Ding, A., and Nathan, C. 1988. Deactivation of macrophages by transforming growth factor-beta. Nature. 334:260-262.

46. Veldhoen, M., Hocking, R.J., Atkins, C.J., Locksley, R.M., and Stockinger, B. 2006. TGFbeta in the context of an inflammatory cytokine milieu supports de novo differentiation of IL-17-producing T cells. Immunity. 24:179-189.

47. Veldhoen, M., and Stockinger, B. 2006. TGFbeta1, a "Jack of all trades": the link with pro-inflam- matory IL-17-producing T cells. Trends Immunol. 27:358-361.

48. Bettelli, E., et al. 2006. Reciprocal development pathways fro the generation of pathogenic effector Th17 and regulatory T cells. Nature. 441:235-238.

49. Mangan, P.R. 2006. Transforming growth factor $-\beta$ induces development of the Th17 lineage. Nature. 441:231-234.

50. Han, J., et al. 2000. Transforming growth factor - $\beta 1$ (TGF- $\beta 1$ ) and TGF- $\beta 2$ decrease expression of CD36, the type B scavenger receptor, through mitogen-activated protein kinase phosphorylation of peroxisome proliferator -activated receptor- $\gamma$. J. Biol. Chem. 275:1241-1246.

51. Tridandapani, S., et al. 2003. TGF- $\beta 1$ suppresses myeloid $\mathrm{F} c \gamma$ receptor function by regulating the expression and function of the common $\gamma$-subunit. J. Immunol. 170:4572-4577.

52. Imai, K., et al. 2000. Transforming growth factor $\beta$ inhibits lipopolysaccharide-stimulated expression of inflammatory cytokines in mouse macrophages through downregulation of activation protein 1 and CD14 receptor expression. Infect. Immun. 68:2418-2423.

53. Garred, P., et al. 2002. Mannose-binding lectin (MBL) therapy in an MBL-deficient patient with severe cystic fibrosis lung disease. Pediatr. Pulmonol. 33:201-207.

54. Anaissie, E. 2007. Intravenous recombinant human mannose-binding lectin (rhMBL) in patients with multiple myeloma receiving chemotherapy followed by stem cell transplant. http://clinicaltrials. gov/ct/show/NCT00388999.

55. Freifeld, A., and Emre, S. 2007. A multicenter study evaluating the safety and tolerability of IV recombinant human mannose binding lectin (rhMBL) in liver transplant recipients. http://clinicaltrials.
gov/ct/show/NCT00415311.

56. Summerfield, J.A. 2003. Clinical potential of mannose-binding lectin-replacement therapy. Biochem. Soc. Trans. 31:770-773.

57. Schluchter, M.D., Konstan, M.W., and Davis, P.B. 2002. Jointly modelling the relationship between survival and pulmonary function in cystic fibrosis patients. Stat. Med. 21:1271-1287.

58. Knudson, R.J., Lebowitz, M.D., Holberg, C.J., and Burrows, B. 1983. Changes in the normal maximal expiratory flow-volume curve with growth and aging. Am. Rev. Respir. Dis. 127:725-734.

59. Corey, M., Levison, H., and Crozier, D. 1976. Fiveto seven-year course of pulmonary function in cystic fibrosis. Am. Rev. Respir. Dis. 114:1085-1092.

60. Cutting, G.R., et al. 1989. Analysis of DNA polymorphism haplotypes linked to the cystic fibrosis locus in North American black and Caucasian families supports the existence of multiple mutations of the cystic fibrosis gene. Am. J. Hum. Genet. 44:307-318.

61. Taylor, J.D., et al. 2001. Flow cytometric platform for high-throughput single nucleotide polymorphism analysis. Biotechniques. 30:661-666, 668-669.

62. Ye, F., et al. 2001. Fluorescent microsphere-based readout technology for multiplexed human single nucleotide polymorphism analysis and bacterial identification. Hum. Mutat. 17:305-316.

63. Livak, K.J., Marmaro, J., and Todd, J.A. 1995. Towards fully automated genome-wide polymorphism screening. Nat. Genet. 9:341-342.

64. Schluchter, M.D., Konstan, M.W., Drumm, M.L., Yankaskas, J.R., and Knowles, M.R. 2006. Classifying severity of cystic fibrosis lung disease using longitudinal pulmonary function data. Am. J. Respir. Crit. Care Med. 174:780-786. 